MATEC Web of Conferences 25, 01007

(2015)

DOI: $10.1051 /$ matecconf/ 20152501007

(c) Owned by the authors, published by EDP Sciences, 2015

\title{
Summary of GOOSE Substation Communication
}

\author{
Fei Xia, Zongze Xia \& Xiaobo Huang \\ State Grid Liaoyang Electric Power Supply Company, Liaoyang, Liaoning, China.
}

\begin{abstract}
The fast real-time application message is an object-oriented substation event-GOOSE (Generic Object Oriented Substation Event). It mainly works on the process layers and bay layers of digital substation. GOOSE intelligent substation communication network can realize the key function of the overall intelligent substation functions. This paper will make a systematic analysis of GOOSE. At first, it will provide a detailed analysis of the transmission mechanism and process of GOOSE message. As certain practical operability lacks the actual application of the multicast filtering method which is applied to GOOSE message based on IEC61850 standard, this paper will summarize three message filtering methods, compare the advantages and disadvantages in topological networks of different kinds in accordance with the instantaneity of GOOSE intelligent substation communication network and draw a conclusion that the independent configuration scheme of GOOSE and SV can adapt to the requirement of times, so as to provide theoretical references for the selection of practical intelligent substation communication network in the future.
\end{abstract}

\section{Keywords: GOOSE; intelligent substation; communication network; IEC61850}

\section{INTRODUCTION}

With rapid development of electric power system, China has realized transformation and updating in old integrated substation automation. What's more, China also has completed the integrated automation in unmanned duty system so as to achieve improved reliability in economical duty and system management. However, as most domestic substations comply with the IEC60870 series treaties ${ }^{[1]}$ that issued in 1997, the system information processing is weak in timeliness. On one hand, the treaties add more workload in integrating substation automation system; on the other hand, the necessity of achieving data transmission through dedicated protocol converter among different devices makes it heavier in point workload. This problem has become the bottleneck in the development of automation communication system. Based on the above background, a new communication protocol standard appeared - the IEC61850 ${ }^{[2]}$ standard. It obtained further extension and development in 2011. Resulting from the IEC61850 standard, intelligent substation has emerged at the right moment. As IEC61850 standard introduces GOOSE via the generic substation status event (GSSE) of UCA and makes GOOSE become one of its core technologies [3], the topological network of GOOSE has become an important part of intelligent substation and played a key role in realizing the functions of the entire intelligent substation system. Thus, there's great significance in studying intelligent substation communication network based on GOOSE.

Content of IEC61850 standard is various, including multiple protocols and technologies. At present, most domestic and overseas document literature can only provide certain understanding of protocol content. Due to the limitation imposed by overseas technologies, domestic application on IEC61850 standard is in continuous practice. However, since GOOSE was introduced into the standard, it has realized P2P communication in reality. The introduction of GOOSE has eliminated the defects that once existed in the non-networked serial connection schemes and master-slave mode. In the meantime, it has helped reduce relative cost ${ }^{[4]}$. Three characteristics of GOOSE which can offer broad development space that have been found in summarizing and concluding related literature: high efficiency in data transmission, realization of function distribution, and realization of real $\mathrm{P} 2 \mathrm{P}$ communication.

However, currently domestic study on GOOSE is not comprehensive. This paper will provide a systematic analysis of GOOSE message transmission mechanism and the structure of GOOSE intelligent substation communication layer network, helping eliminate the defects in this area. According to the timeliness of GOOSE intelligent substation communication network, this paper will compare relative merits of different topological networks and provide theoretical references for the selection of practical intelligent substation communication network in the future.

\section{ANALYSIS OF GOOSE MESSAGE TRANS- MISSION AND PROCESS}

\subsection{GOOSE message transmission mechanism}

GOOSE refers to a request mechanism of the standard which accords with the substation automation system fast message. The generally generic object-oriented

This is an Open Access article distributed under the terms of the Creative Commons Attribution License 4.0, which permits unrestricted use, distribution, and reproduction in any medium, provided the original work is properly cited. 


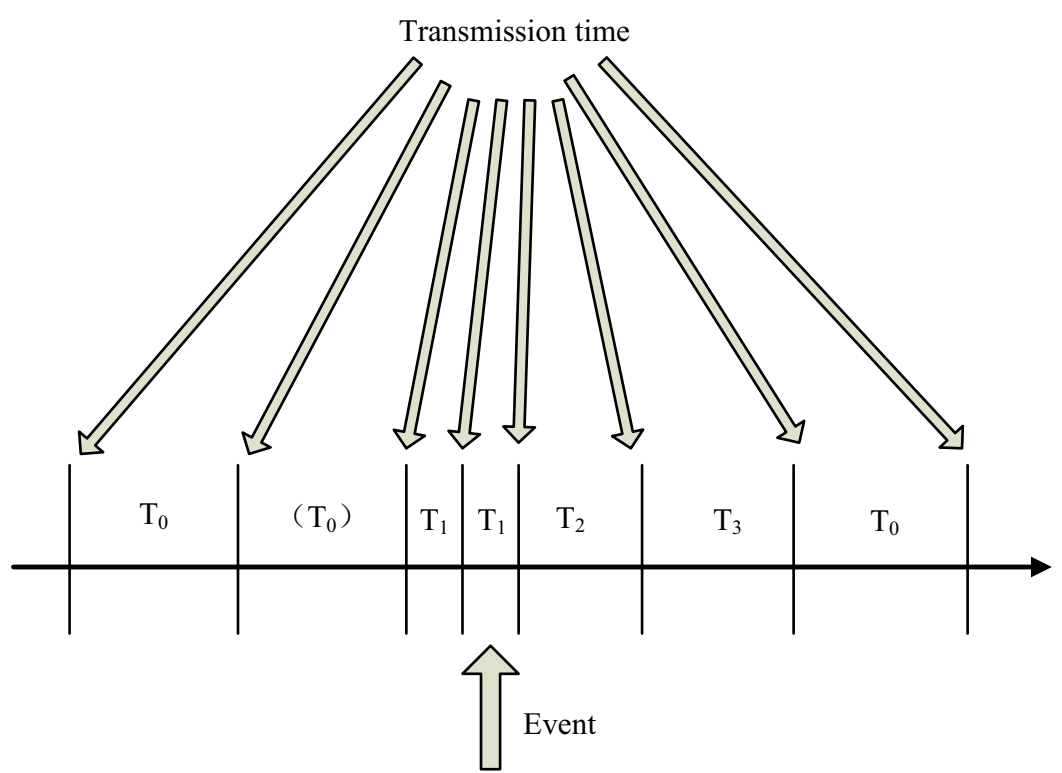

$\mathrm{T}_{0}$ : The stable time retransmission

$\left(\mathrm{T}_{0}\right)$ : The time of stabilization may be shortened

$\mathrm{T}_{1}$ : The shortest transmission time

$\mathrm{T}_{2}, \mathrm{~T}_{3}$ : The retransmission time under the condition of stability directly

Figure 1. Event transmission time

GOOSE means the messages which can share and publish any information of IEC61850. The first version of IEC61850 kept generic substation status event (GSSE). However, GSSE was unbonded to IEC61850 system and had no actual application in practice. As a result, GSSE was deleted in the second version ${ }^{[5]}$ Compared with GSSE, GOOSE contains better flexibility. In general, the transmission objects of GOOSE come from the data set of each logic node.

In order to guarantee the reliability and timeliness of GOOSE, the sequence retransmission mechanism based on Ethernet multicast technology is applied in GOOSE message. Compared with traditional manufacturing message specification (MMS), GOOSE contains two outstanding features: One is its superior timeliness and the high efficiency can meet the requirements set in related information transmission of relay protection; the other one is that its one-transmitting and multi-receiving feature is able to share disconnectors, voltage and current.

GOOSE transmits protocols by realizing cycles of Ethernet multicast addresses: When data stays the same, transmission can rapidly occur three times within one cycle. GOOSE may also transmit the entire data in one long cycle as shown in the figure given below (Figure 1).

$\mathrm{T}_{0}$ refers to the interval in long-time stable transmission. Once any change occurs in data, data updating and repeated transmission will happen regardless of the transmission mode of the previous frame. The fastest retransmission interval and heartbeat interval can be defined. If $T_{0}$ is $5 \mathrm{~S}$ and $\mathrm{T}_{1}$ is $1 \mathrm{~ms}$, the associated interlock applications corresponding to the intervals can be extended to $10 \mathrm{~s}$ and $100 \mathrm{~ms}$. $\mathrm{T}_{2}$ and $\mathrm{T}_{3}$ refer to the retransmission time in the process from fluctuant state to stable state. No specific definition is given for this period. In general, successive doubling exponential algorithm is commonly used in engineering practice $^{[6]}$.

\subsection{Analysis of GOOSE message process}

If any value change appears in any member of GOOSE message, the entire system will send GOOSE message. Usually, GOOSE message can be divided into APDU and frame header (FH). As a rule, Ethernet type is often used as the basis to define $0 \times 88 \mathrm{~B} 8$ in GOOSE message. However, as the TPID value is defined as $0 \times 8100$ in Tag, GOOSE message processing is required. There are two values which can be regarded as the basis to make judgment. See Figure 2 given below for the corresponding processing flow.

Part data forms in APDU are very special. Data in APDU can be displayed in form of "data + data length + identification" after being coded through GOOSE PDU. In this form, the total data length is not fixed and the identification sequence is as follows: $0 \times 80$, data set $0 \times 81$, structural body $0 \times 82$, Boolean type $0 \times 80$, bit string $0 \times 84$, integer $0 \times 85$, unsigned integer 0x86, floating point type $0 \times 87,0 \times 88$, octet, and $0 \times 8 \mathrm{~A}$. 


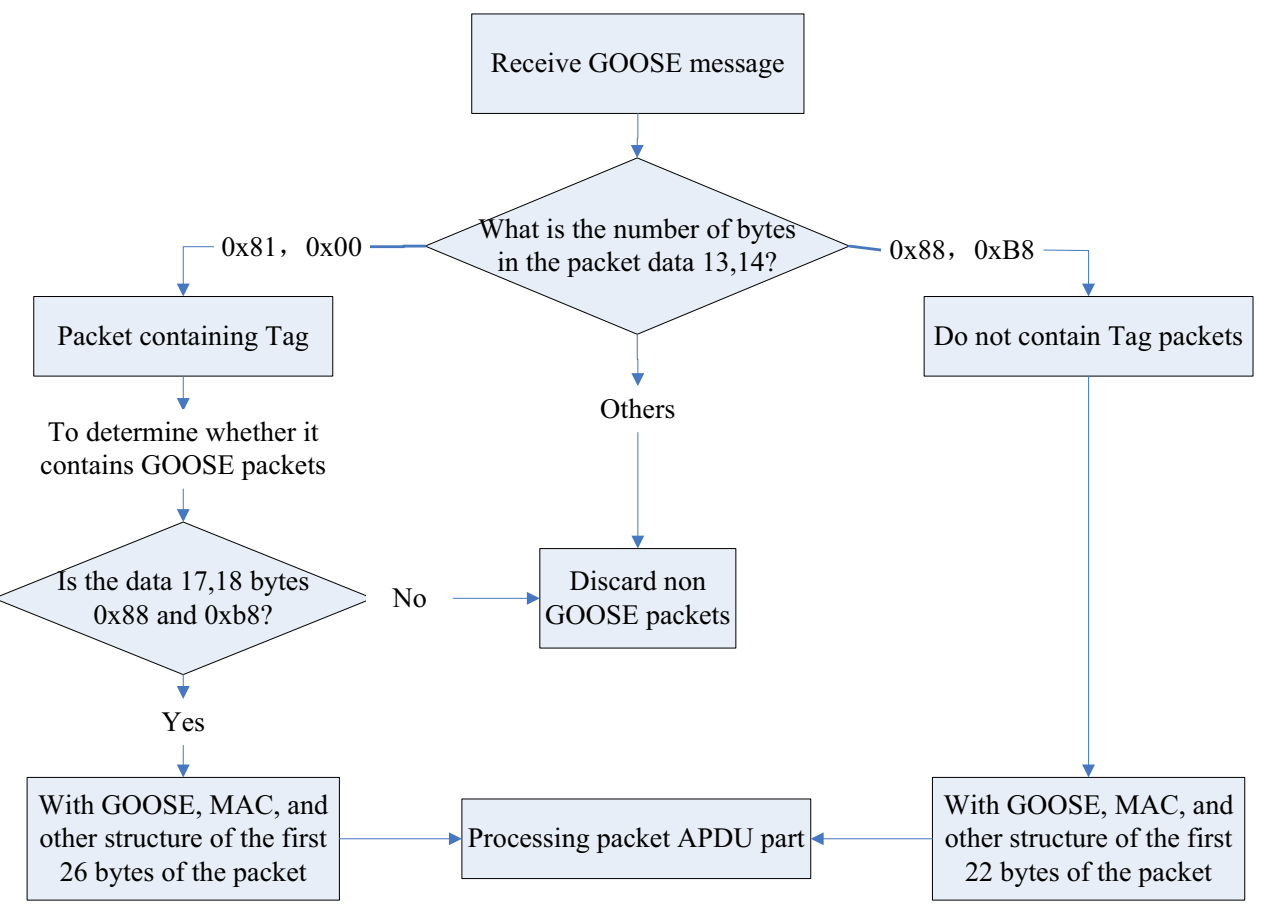

Figure 2. GOOSE message processing flow

The location pointer can be defined through analysis. Analyze data members according to the data length and data identification. Thereafter, conduct an analysis of the data columns in corresponding locations by data degrees. Move the pointer and analyze the next column until the enter data column is analyzed.

\section{GOOSE MESSAGE FILTERING METHODS}

GOOSE of IEC61850 standard is a multicast communication mechanism to solve the problems existing in IED. In practical operation process, multicast filtering methods have no certain operability. Based on this, this paper will compare and analyze the three GOOSE message filtering methods shown as follows.

(1) Set-up of IED network card multicast MAC address filtering message

In order to strengthen the overall receiving performance of multicast message, the best way is to apply MAC hardware filtering. After the IED network card receives multicast message, we should decide whether to set up the group member in accordance with the installed program. "No" refers to waive and "Yes" refers to accept.

Although network card multicast MAC address filtering method cannot solve the excessive spreading problem of network GOOSE message, it can reduce unnecessary occupation of IEC in CPU resource. Yet, it is hard to manage the algorithm of each manufacturer. As a result, there's certain filtering "leak" in network multicast MAC address filtering method. Besides, while expanding substation address, the entire system may reset the distribution of multicast address, and thus may remodify the configuration of operational equipment. Therefore, during the process of network card MAC address filtering, it is impractical to integrate devices from multiple manufacturers into substation automation system in practice.

(2) Switch multicast filtering

This method can be divided into two types: the static multicast distribution and the dynamic multicast distribution.

The static multicast distribution is easy in operation. However, as the switch port connected with IED is fixed, it is complicated to set up the port. When malfunction occurs, both switch and its configuration must be replaced at the same time, which is dangerous to operate.

An important precondition of dynamic multicast distribution is both IED and switch shall support the two-layer multicast protocol at the same time. At present, there are two multicast protocols to reach this goal: GMRP protocol and IGMP snooping protocol. GMRP protocol is mainly used for multicast registration information. The main operating principle is as follows: When one host computer sends GMRP information, switch will place it inside the port and further transmit the information in VLAN to make sure the multicast source can receive it. When switch sends the multicast message to the port, it can realize two-layer multicast transmission. IGMP snooping protocol is 


\section{MATEC Web of Conferences}

mainly used in managing and controlling multicast groups. The main operating principle is as follows: IGMP message will be analyzed at first. When switch receives the IGMP information, the host computer will store the information in a multicast table. When it receives the message to leave, it will delete the stored information. Two-layer multicast transmission can be achieved in this way. By analyzing these two protocols, it can be seen that although there's certain limitation in GOOSE communication path to make it more appropriate, the protocol will be seldom applied in practical operating process. Therefore, IGMP snooping protocol can realize two-layer multicast transmission with most switches and it is more commonly used in practice.

Compared with the static multicast protocol, the dynamic multicast protocol can simplify the configuration to a large extent and classify multicast message. However, as IED and IEC61850 standard cannot support the dynamic multicast protocol, the static multicast distribution is more commonly applied.

(3)Switch VLAN

There are three classification methods for this means: the method based on MAC address, the method based on protocol and the method based on port.

1. The method based on MAC address is complicated in initial configuration. However, it can solve the problem of replacing mobile devices in a better way.

2. The method based on protocol makes classification according to network address. In operation, switches which can bear three-layer switching must be selected.

3. The method based on port is the easiest one to master. However, it is hard to solve problems such as replacing mobile devices by this means.

As there is no three-layer message structure or need of replacing mobile devices in GOOSE message, it can be seen that the third one, namely the method based on port, is the best way to divide VLAN. In order to fundamentally avoid the influence that invalid GOOSE message may leave on IED, classify VLAN to separate invalid GOOSE message. Certainly, more VLAN will be required for substation with large data scale. Although this method can separate invalid message to a certain degree, mistakes may frequently occur in operation due to its complicated configuration.

Based on the above analysis, it can be concluded that the static set-up switch multicast filtering is more applicable for the GOOSE message of IEC61850 standard.

\section{STRUCTURE OF GOOSE INTELLIGENT SUBSTATION COMMUNICATION LAYER NETWORK}

\subsection{Comparison of GOOSE intelligent substation communication layer network message types and forms}

According to related regulations specified in IEC61850 standard and different needs in data trans- mission of substation, there are seven commonly used message forms ${ }^{[8]}$ :

\section{Time synchronization message}

According to different time alignment methods, the time synchronization message is mainly used for the internal clock synchronization of intelligent equipment Different levels of time synchronization precision requirements can be met by pulse or difference.

\section{File transmission message}

The file transmission message is mainly used in file transmission, such as large-scale data files of set point protection, waveform records and information data.

3. Raw data message

The raw data message includes transmission data between transmitter and mutual inductor. In situation when mutual inductor technology is not required, the raw data message can be constituted by the synchronized data stream formed by each device.

4. Low-speed message

The low-speed message requires display of system parameters, constant values of reading/rewriting installations, and transmission event records.

5. Intermediate-speed message

The transmission time of intermediate-speed message is relatively short. This message form includes status information under normal operation and clock information of equipment.

6. Trip-status message

As trip-status message contains features of fast message in substation, requirements for timeliness in this form are high.

7. Fast message

The fast message includes binary coding of simple message, data, and orders, such as switch change, removal, start-up, reclosing, primary interlocking of tripping and closing.

By comparison and analysis, it can be seen that the time synchronization message is mainly applied in intelligent equipment or in situation when high precision is needed. The file transmission message is mainly applied for large-scale data transmission. The raw data message is mainly applied in synchronized data transmission of multiple devices. The low-speed message, intermediate-speed message and fast message are mainly applied for needs in different data transmission speeds. The trip-status message shall be used when requirement for timeliness is high. These seven message forms all have their own features and can be used in different data transmission conditions. Users can make choices according to their actual needs.

In order to optimize the decoding process of receiving message, GOOSE message directly uses specific Ethernet type. Message mapping ${ }^{[9]}$ is shown in Figure 3 given below.

The complete message transmission process includes the processing methods of sender and receiver. The period for sender to deliver data to receiver is the transmission time which is also the sum of network transmission delay and message delivery delay of physical device 1 and physical device 2 . See the process in Figure 4 given below. 


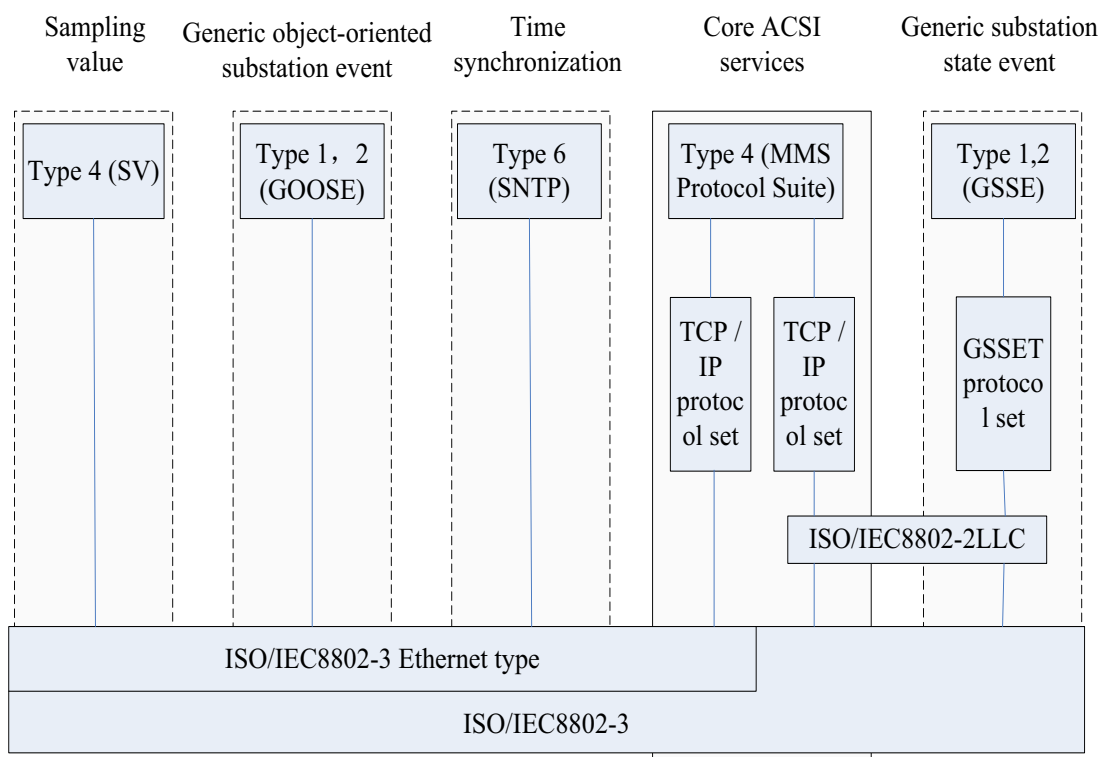

Figure 3. Protocol suite and functional diagram

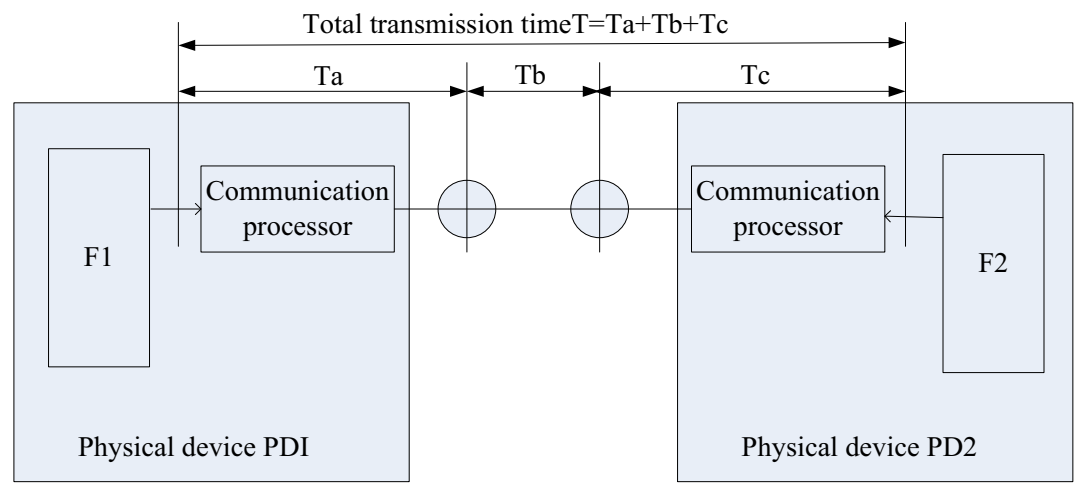

Figure 4. Definition of total transmission time

The transmission delay between these two physical devise are composed of various components in the figure. GOOSE transmission delay occurs in this process.

(1) Delivery delay

The message delivery delay refers to message processing delay of device communication processor. It is mainly tested in pilot project of State Grid Corporation of China (SGCC). Delay the processing of each port for about 20 microseconds and calculate the second endpoint. The rest can be done in the same manner.

(2) Output delay

The network transmission delay refers to the total time used by message sender. Data frame can cause certain delay inside switch. The total network transmission delay includes frame queuing delay, circuit transmission delay, switch delay and delivery delay.
For a 100 trillion star-topology Ethernet network, the transmission delay of a SV is $20-160$ microseconds while that of a GOOSE frame is about 70 microseconds.

\subsection{Comparison of GOOSE message communication} network structures

GOOSE message mainly completes signal transmission through the process network inside substation. In intelligent substation communication network, the main topological structures of communication network are respectively the bus-topology structure, the loop-topology structure and the star-topology structure [10].

(1) Bus-topology structure

Bus-topology structure means that the bus of each node is directly connected with the knot of hardware 
MATEC Web of Conferences

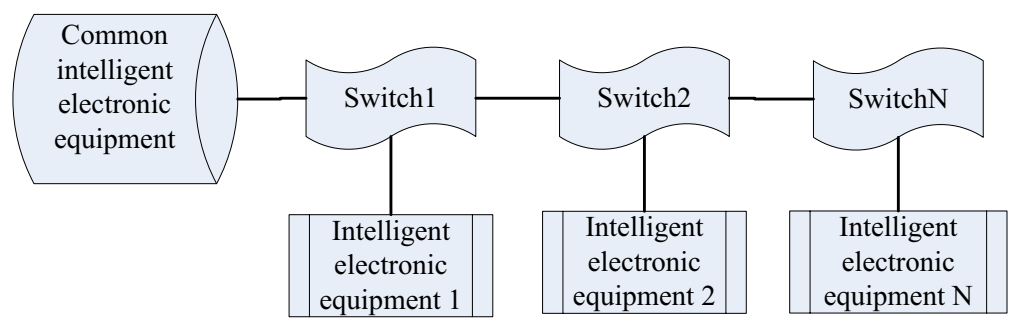

Figure 5. Bus-topology

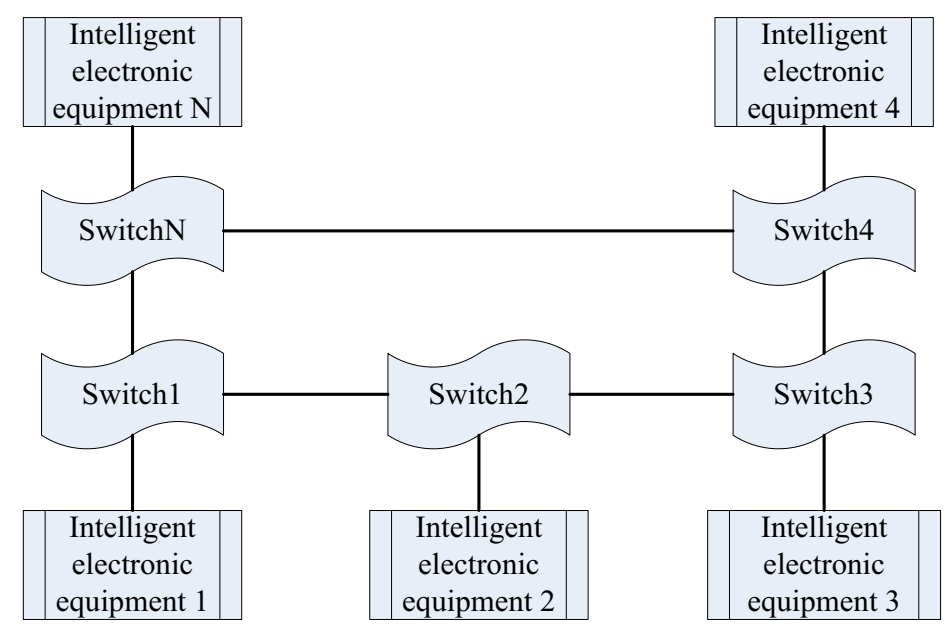

Figure 6. Loop-topology

as shown in Figure 5. Its main advantages are as follows: It is easy to realize expansion between the connecting nodes of network and intelligent equipment; the wiring arrangement is simple, and the cost is low. There are certain disadvantages as well:

1. The contained transmission delay is not applicable for situation when high timeliness is required.

2. It's hard to diagnose or separate when malfunction occurs.

3. Bus failure will leave serious influence on system which can easily cause bottleneck effect.

(2) Loop-topology structure

The loop-topology structure refers to the loop structure which is connected by each switch. In this structure, the operation of the entire network structure will not be affected no matter which node has malfunction. See Figure 7 shown below for details. The main advantage of this structure is that it has good network redundancy performance. The network can adjust itself to make the entire network work normally regardless of malfunction location. The disadvantages are shown as follows:

1. There is broadcast storm risk.

2. The message delay is not stable.

3. There is a great difficulty in expansion. New switch can only be added and grouped after opening the original network which will result in higher cost.

4. There is limitation in switch quantity.

5. The network protocol and structure are complicated.

6. Timeliness is weak. When malfunction occurs, the self-adjustment time cannot meet network communication requirements.

(3) Star-topology structure

The center of star-topology structure is the public switch. This structure is formed by connection which is composed of layers. Therefore, the star-topology structure can help shorten the waiting time and reduce the delay time. The main advantages are shown in Figure 7 given below:

1. It can eliminate the potential risk of network storm.

2. The network delay is less while the message delay is steady, and they are able to meet normal network communication requirements.

3 . For any two computers, three switches are required at most. The speed is high.

4. If any malfunction occurs in the center switch, no interval switch will be influenced.

5. If any malfunction occurs in any switch, the switch can be individually isolated without leaving any influence on the others. 


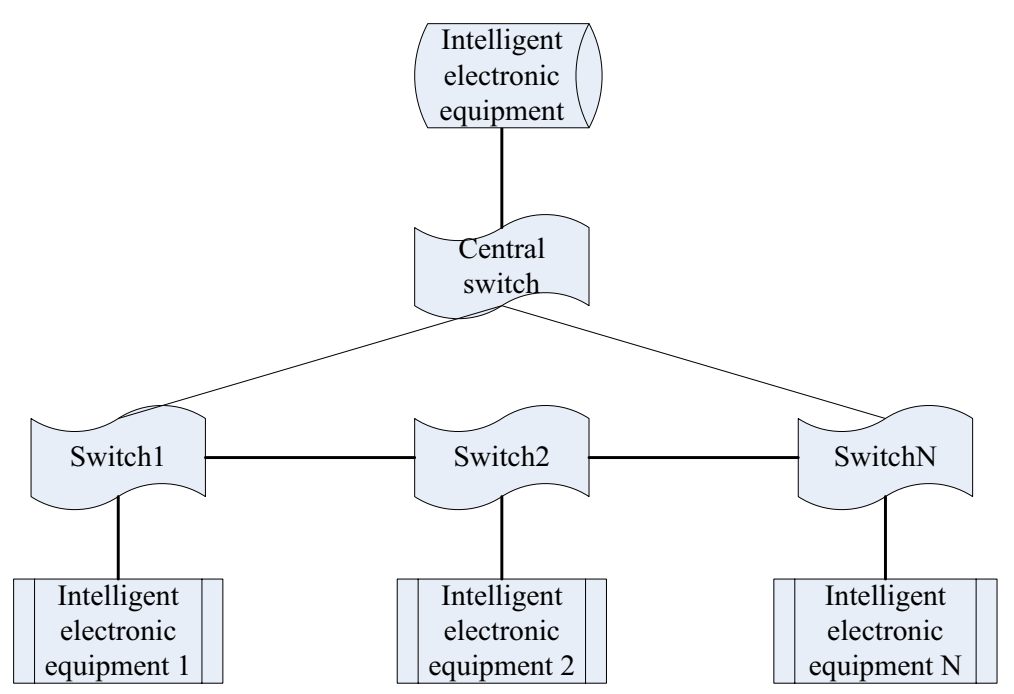

Figure 7. Star-topology

6. It's convenient for expanding and easy in wiring arrangement.

7. The timeliness is good.

The disadvantage of star-topology structure is network redundancy which won't be good when the load of center switch is excessive in overhauling.

Based on the above comparison and analysis, it can be concluded that the star-topology structure has more advantages in GOOSE network data transmission. As the time of operation and intelligent substation production is short while the obtained experience is not enough at present, the process-layer networking scheme is more commonly-used. The main networking schemes are as follows:

(1) Use straight-collecting \& straight-tripping scheme with no configuration in the process-layer network. The disadvantages are as follows: The unvarnished transmission function of space-layer device will be used which can increase processing pressure; and quality requirements for the switches of station-level websites will be high. The advantages are as follows: The investment cost is low; the optical fibers are clear; the operation is convenient; the process-layer network can be cancelled.

(2) Common network transmission of time synchronization message for IEC61588, GOOSE and SV. It can protect the straight-collecting and straight-tripping mechanism. The disadvantages are as follows: Switches which can support this scheme are high in price and the related operating experience is little. The advantages are as follows: The switch data is little; maintenance is convenient; the network structure is simple.

(3) GOOSE networking with point-to-point SV form. It can protect the straight-collecting and straight-tripping mechanism. The disadvantages are as follows: Wiring of sampling values is complicated; data cannot be directly shared; realization of functions is hard. The advantages are as follows: The switch quantity is small and the network tripping can save optical cables.

(4) Common transmission of GOOSE and SV message. It can protect the straight-collecting and straight-tripping mechanism. The disadvantages are as follows: The required optical cables are excessive; information cannot be shared and it is not convenient for failure analysis; calorific values of its multi-light ports are high and they will shorten the service life; the quantity of linking points is large, which will make wiring complicated; the communication pressure of equipment is high, which will cause certain delay in message processing; the quantity of optical fiber port is large. The advantages are as follows: The quantity of required switch is small; the direct tripping can be protected; no switch will be relied on.

(5) Individual configuration of GOOSE and SV network. It can protect the straight-collecting and straight-tripping mechanism. The disadvantages are as follows: The required switches are excessive; the requirement for optical cable is high; the information cannot be shared; the quantity of linking points is large which will make wiring complicated; the communication pressure of equipment is high, which will cause certain delay in message processing. The advantages are as follows: The networking of GOOSE and SV can ensure no influence will be left on message transmission; the straight-collecting and straight-tripping mechanism can be protected; no switch will be relied on.

The following conclusion can be reached based on the above analysis: In order to maintain the speed and reliability of relay protection tripping, and in consideration of the current shortage in network operation, the straight-collecting and straight-tripping scheme individually set up from GOOSE and SV is more applicable at the present stage. 


\section{MATEC Web of Conferences}

\section{CONCLUSION}

This paper has made a systematic study on GOOSE intelligent substation communication network which plays a key role in realizing the functions of the entire intelligent substation. It explains the definition, features, message and message analysis approach of "GOOSE" at first; then, it studies GOOSE message process and concludes the detailed processing flow in practical application. By comparing the three filtering methods of GOOSE message and based on the current situation of network equipment and IED, this paper draws the conclusion that static-allocation switch multicast filtering method is the best solution.

This paper has made a systematic analysis on the structure of GOOSE intelligent substation communication layer network. By analyzing the timeliness of GOOSE intelligent substation communication layer network and comparing it with topological networks of different types, it has found the advantages and disadvantages. By comparing various common network forms and making related analysis, this paper has drawn the conclusion that GOOSE network has certain advantages in message transmission and network allocation. It can provide theoretical references for the selection of practical intelligent substation communication network in the future.

\section{REFERENCES}

[1] IEC61850-1, Communication Network and System in Substations-Part 1: Introduction and overview[S]. Geneva: IEC, 2003.

[2] IEC61850-9-1, Communication networks and systems in substation-Part 9-1: Specific communication service mapping (SCSM)-Sampled values over serial unidirectional multidrop point to point link[S], Geneva: IEC, 2003.

[3] JIEC61850-9-2, Communication networks and systems in substation-Part 9-2: Specific Communication service mapping (SCSM)-Sampled values over ISO/IEC 8802-3[S]. Geneva: IEC, 2004.

[4] He, L. \& Zhan, W. 2010. Problem Analysis of GOOSE Technology in Substation Application. Hebei Electric Power, 29(4):2-4.

[5] Wu, Q.F. \& Chen, K. IEC61850 and Applied Research on Digital Substation. Electrical Technology, 2009, 62(2): 5-11.

[6] Li, J., Xue, T. \& Zhu. B. 2012. Brief Analysis of the Framework and Primary Technology of Intelligent Substation. Discovering Value, 25(7): 22-33.

[7] Wu, Y.C. \& Wang, Z.P., et al. 2009. Application and Analysis of Substation Configuration Language. Electric Power System Protection and Control, 37(15): 18-22.
[8] Wang, Z.Z. \& Sun, D. 2011. Study on IEC61850 Configuration File Test. Electric Power System Protection and Control, 39(6): 8-12.

[9] Jing, L.C., Li, Q.W. \& Dong, F. 2012. Investigation on the Construction of GOOSE Information Model Based on IEC61850 Standard. Automation \& Instrumentation, 164(6): 10-15.

[10]Gao, Y.D., Zhu, B.S. \& Li, H. 2011. Research on the Application of the "Virtual Terminator" Design Method of Digital Substation. Electric Power System Protection and Control, 39(5): 15-20.

[11]Chen, H.D., Yun, B.J. \& Dai, W.Z., et al. 2013. Study of the Switching Station Protection Based on GOOSE Mechanism. Electric Power System Protection and Control, 41(13): 120-136.

[12]Yang, W.Z. 2014. Reliability Analysis of Digital Substation Based on Bayesian Network. Architectural Engineering Technology and Design, (4): 540-561.

[13]Hu, J.B., Chang, X.J. \& Huang, R.H. et al. 2011. "Three-in-One" Netowrking Scheme and VLAN Division of Intelligent Substation Process Level. Electgrotechnics, 39(9): 9-14.

[14]FAN Chen, NI Yi-min, \& DOU Ren-hui, et al. 2011 Analysis of networkscheme for process layer in smart substation. Automation of Electric Power Systems, 35(18): 64-70.

[15]Huang, S.X., Huang, T.G. \& Cheng, X. 2012. Study of the Information Integration Platform Construction Scheme of Intelligent Substation. Northeast Electric Power Technology, 33 (2): 19-25.

[16]Fan, C., Li, Y.M. \& Dou, R.H., et al. 2011. Analysis of Intelligent Substation Process Level Networking Scheme. Power System Automation, 35(18): 64-70.

[17]Zhang, Y.L., Chen, X.Q. \& Wang, C.M., et al. 2012. Reliability Assessment on the Second-order System of Intelligent Substation. Power System and Clean Energy, 28(11):6-13.

[18]Zhu, Q.C., Su, J. \& Zhao, Y.H., et al. 2012. Analysis and Study of the Three-in-One Network Architecture of Intelligent Substation. Mechanical and Electrical Information, 56(36): 141-146

[19]Cao, H.O., Yan, G.P. \& Xu, N., et al. 2011. GOOSE Networking Scheme of Digital Substation. Electric Power Automation Equipment, 31(4): 142-154.

[20] Yang, G., Wang, Z.Q. \& Wang, W.L., et al. 2012. Discussion about the Key Technology of Intelligent Substation Process-Level Switch. Electrical Technology, (1) 49-56.

[21]Ren, Y.M. \& Cao, F.M. 2013. New Trend and New Application of IEC61850. Power System Automation, 37(2): 2-7.

[22] CUI Quan-sheng, WEI Yong, \& HE Yong-ji, et al. 2011. Analysis of PTP1588 protocol. Power System Protection and Control, 39 (10): 140-152. 